\title{
Bipolar mood disorder and treatment-resistant depression
}

\author{
Janusz K Rybakowski \\ From $1^{\text {st }}$ International Congress on Neurobiology and Clinical Psychopharmacology and European \\ Psychiatric Association Conference on Treatment Guidance \\ Thessaloniki, Greece. 19-22 November 2009
}

Two lines of evidence point to the relationship between bipolar mood disorder and treatment-resistant depression. First, the frequency of suboptimal response to antidepressant drugs is significantly higher in bipolar than in unipolar mood disorder. Secondly, the first and the second generation mood stabilizing drugs are efficacious for augmentation of antidepressants in treatmentresistant depression.

Short-term non-response to antidepressant drugs has been significantly higher in bipolar and in unipolar mood disorder. A significant percentage of unipolar depressed patients resistant to antidepressant drugs reaches a diagnosis of bipolarity during a specific screening or during follow-up. In the Polish all-country DEP-BI study including 880 depressed outpatients treated with psychiatrists, it was found that the percentage of treatment-resistant depression was significantly higher in bipolar than in unipolar mood disorder, especially so, in bipolar spectrum disorder where the features of bipolarity are most easy to overlook. Recent Polish TRESDEP study included 1051 depressed patients (569 with treatment-resistant and 482 with treatment non-resistant depression). Using Hypomania Check List-32 (HCL-32) scale, it has been found that the percentage of $\geq 14$ score on HCL-32, indicating bipolarity, was significantly higher in patients with treatment resistant than in treatment-nonresistant depression $(44 \%$ vs $30 \%$, respectively).

Lithium, the prototypic mood stabilizer, has been the most frequent modality used for the augmentation of antidepressants in treatment-resistant depression, and the efficacy of lithium in this respect has been confirmed by a number of meta-analyses. Our study has shown that such effect of lithium is significantly better

Department of Adult Psychiatry, Poznan University of Medical Sciences, Poland in bipolar than in unipolar depression. Out of the first generation antidepressant drugs, also some efficacy of carbamazepine, and to less extent, valproate, has been found for the augmentation of antidepressants. Lamotrigine, new generation anticonvulsant and mood-stabilizer, demonstrated its antidepressant efficacy both during acute episode and in prophylaxis of depression in bipolar illness. It has been also effective for augmentation of antidepressant drugs in treatment-resistant depression. In our study we found that the overall efficacy of lamotrigine was similar to that of lithium, however, both drugs were more efficacious for augmentation of venlafaxine than paroxetine.

In recent years, there have been numerous studies on the efficacy of second generation antipsychotic drugs (some of them can be qualified also as second generation mood stabilizers) for the augmentation of antidepressants in treatment-resistant depression. While the clear antidepressant effect of quetiapine as monotherapy has been demonstrated, the majority of second generation antipsychotics proved their efficacy in augmentation of antidepressants in treatment-resistant depression, and aripirazole, obtained an official approval for such indication.

Published: 22 April 2010

doi:10.1186/1744-859X-9-S1-S14

Cite this article as: Rybakowski: Bipolar mood disorder and treatment-

resistant depression. Annals of General Psychiatry 2010 9(Suppl 1):S14. 